\title{
MANUTENÇÃO DA CAPACIDADE INOVADORA NA EXTERNALIZAÇÃO DO DESENVOLVIMENTO DE PRODUTOS: O CASO DA INDÚSTRIA AUTOMOBILÍSTICA
}

\section{INNOVATIVE CAPACITY MAINTENANCE BY AUTOMAKERS IN A PRODUCT DEVELOPMENT OUTSOURCING SCENARIO - THE CASE OF BRAZILIAN AUTOMOTIVE INDUSTRY}

\author{
Adriana Marotti de Mello'; Roberto Marx ${ }^{2}$ \\ ${ }^{1}$ Escola Politécnica da Universidade de São Paulo - Brasil adriana.mello@poli.usp.br \\ ${ }^{2}$ Escola Politécnica da Universidade de São Paulo - Brasil robemarx@usp.br
}

\begin{abstract}
Resumo
Com o objetivo de diversificar o portfolio de produtos e reduzir prazos e custos de desenvolvimento, cada vez mais as empresas externalizam o Desenvolvimento de Produtos (DP) para fornecedores. Se por um lado, o maior envolvimento de fornecedores garante acesso a novas tecnologias, por outro lado pode impactar negativamente na capacidade inovadora da empresa, através da dependência do conhecimento de seus fornecedores. Então, como poderia uma empresa manter sua capacidade inovadora mesmo com a externalização do DP? Este artigo tem como objetivo discutir essa questão, analisando a influência do conhecimento de arquitetura, ou seja, o conhecimento de como integrar diferentes peças e módulos em um sistema com funcionalidade integrada, na manutenção da capacidade inovadora em uma empresa que externaliza atividades de DP para seus fornecedores. Foi realizado em estudo de caso sobre o desenvolvimento de um veículo popular no Brasil, e as conclusões apontam que a empresa que mantém controle sobre o conhecimento de arquitetura do produto, poderia manter sua capacidade de inovar, mesmo com a externalização do DP.
\end{abstract}

Palavras-chave: Capacidade Inovadora; Desenvolvimento de produtos; Indústria automobilística.

\section{Introdução}

No atual cenário, o Desenvolvimento de Novos Produtos (DP) tem fundamental importância estratégica na definição da competitividade dentro da indústria. Há a necessidade de encurtar prazos de desenvolvimento, diversificar o portfolio de produtos e reduzir custos, como formas de buscar vantagem competitiva sustentável. 
Diferentes empresas em diferentes setores procuraram formas alternativas de organizar o gerenciamento da produção e o desenvolvimento de produtos (DP), mas uma das formas de organizá-los que parece emergir com muita força nos últimos anos, é a externalização de atividades para fornecedores (outsourcing) (CLARK e FUJIMOTO, 1991). A decisão de externalizar determinada atividade é também uma decisão sobre propriedade e acesso ao conhecimento necessário para executar tal atividade, ou seja, externalizando o DP, se dispersa o conhecimento entre diferentes empresas da cadeia de fornecimento (BECKER e ZIRPOLI, 2003, GRAZIADIO e ZILBOVICIUS, 2003).

A dispersão do conhecimento pode afetar a capacidade inovadora de duas maneiras: se por um lado, haveria uma possibilidade de ganhos com os novos desenvolvimentos oriundos da contribuição dos fornecedores (VON HIPPEL,1988), por outro lado, em longo prazo, poderia significar dependência destes (FINE e WHITNEY, 1996) e, até mesmo, esvaziamento (“hollowing out”) da base de conhecimento da empresa que externaliza o DP (BECKER e ZIRPOLI, 2003).

Chega-se então, a um dilema: como uma empresa pode usufruir os benefícios da externalização do DP mantendo sua capacidade inovadora? Pois, se externalizar o DP pode trazer vantagens para a empresa que o externaliza, em termos de custos e prazos de desenvolvimento e de acesso a novas tecnologias, também pode causar impactos negativos, principalmente quanto à capacidade de inovação, através do esvaziamento da sua base de conhecimento e dependência do conhecimento tecnológico de seus fornecedores.

A solução estaria no domínio do conhecimento de arquitetura, por parte da empresa que externaliza o DP, ou seja, do conhecimento de como integrar diferentes componentes em um sistema coerente (HENDERSON e CLARK, 1990). Esse conhecimento dependeria da empresa possuir conhecimento específico sobre a tecnologia dos componentes de seus produtos e, principalmente, do domínio das especificações das interfaces entre esses componentes (TAKEISHI, 2002). A empresa que domina o conhecimento de arquitetura do produto manteria sua capacidade de inovar dentro dessa mesma arquitetura.

O conhecimento de arquitetura seria de importância fundamental para a capacidade de inovar em automóveis, uma vez que a percepção dos clientes do que é realmente inovador é ligado mais à funcionalidade do conjunto do que da funcionalidade de módulos e componentes em separado (FIXSON e SAKO, 2001).

Este artigo tem por objetivo discutir a questão de como o domínio do conhecimento de arquitetura interfere na manutenção da capacidade inovadora das empresas, através de pesquisa bibliográfica sobre o tema e pesquisa empírica sobre o processo de desenvolvimento do VW Fox, especialmente no que se refere a estratégia de envolvimento de fornecedores. Foi também 
pesquisado o processo de desenvolvimento de dois módulos: o cockpit e os bancos, com o objetivo de aprofundar a pesquisa e incluir a visão dos fornecedores sobre o assunto.

O presente artigo está organizado da seguinte maneira: na Seção 2, apresenta-se a revisão bibligráfica realizada sobre o conceito de conhecimento de arquitetura e qual a influência deste conceito na manutenção da capacidade inovadora de uma empresa, mesmo com a externalização de atividades de desenvolvimento; a Seção 3 traz a metodologia de pesquisa; na Seção 4, são apresentados os resultados da pesquisa empírica realizada e na Seção 5, discutem-se os resultados e traz-se a conclusão do trabalho.

\section{Domínio do Conhecimento de Arquitetura como forma de manter a Capacidade Inovadora de uma empresa}

A diferença entre um produto visto como um sistema e um produto visto como um conjunto de componentes ilustra a idéia dos dois tipos de conhecimento requeridos no DP: o conhecimento de componentes e o conhecimento de arquitetura. O primeiro é o conhecimento dos conceitos básicos de design do produto e de como estes conceitos são implementados em cada componente em particular.

Pode-se definir conhecimento de arquitetura como:

"O conhecimento de como os diferentes componentes são integrados formando um conjunto coerente, um sistema com funcionalidade integrada, e não simplesmente um conjunto de componentes...” (HENDERSON e CLARK, 1990).

O conhecimento de arquitetura surge, portanto, depois que uma organização desenvolveu familiaridade suficiente com a interação dos diferentes componentes que permita que ela possa fragmentar um produto em elementos menores sem que com isso, perca informação crítica da funcionalidade do produto como um todo. Esse conceito de conhecimento de arquitetura é fundamental para se entender o papel da empresa que integra diferentes módulos em um produto com funcionalidade única e foi criado por Henderson e Clark (1990).

Nos estágios iniciais da evolução tecnológica de um setor, há grande experimentação, com diferentes soluções tecnológicas para um mesmo produto. Uma vez que um conceito de produto é estabelecido como dominante, o conjunto inicial de componentes é refinado e elaborado e o progresso tecnológico caminha na direção do desenvolvimento do conceito de cada componente individualmente dentro de uma arquitetura estável, em torno da qual é organizado o setor. Por exemplo, no início do desenvolvimento dos automóveis, foram desenvolvidas diferentes 
motorizações (usando gasolina, eletricidade ou vapor como combustível) e diferentes soluções de tração e carroceria (metal ou madeira). Após a consolidação do conceito dominante de veículo carroceria metálica, com motor de combustão interna e tração traseira - o desenvolvimento do setor foi no sentido de refinar a concepção tecnológica dos componentes dessa arquitetura (HENDERSON e CLARK, 1990).

O conhecimento de arquitetura está diretamente ligado à capacidade de uma empresa de identificar diferentes necessidades funcionais e traduzi-las em especificações técnicas, alocando essas funções nos diferentes módulos que compõe o produto e especificando as interfaces entre esses módulos (FIXSON e SAKO, 2001).

Em um produto com arquitetura modular, mas com funções dispersas por vários módulos ou sistemas, é fundamental a presença de um integrador desses módulos, um coordenador que domine o conhecimento de arquitetura, que defina e ajuste as regras do projeto que determinam interconexões entre as interfaces dos módulos. Nesse ajuste, que muitas vezes ocorre de forma iterativa, o conhecimento dos módulos e seus componentes é necessário. Um exemplo disto é a especificação máxima de ruído de vibração em um automóvel (NVH) em diferentes velocidades. Para conseguir atingir essa especificação, é necessário conhecimento das inter-relações entre a carroceria, o chassis e o conjunto motriz do veículo (SAKO, 2002).

O domínio do conhecimento de arquitetura pode ser definido como a "raison d'être" das montadoras de automóveis e a principal fonte de sua vantagem competitiva (TAKEISHI, 2002). É este domínio que dá às montadoras a capacidade de identificar as necessidades e desejos dos clientes, transformando-a em especificações funcionais de produtos. Perdendo o controle sobre esse conhecimento, poder-se-ia afirmar que uma empresa perderia sua capacidade de inovar. É o que ocorreu com a IBM, no desenvolvimento do PC: ao dotar o produto de uma arquitetura modular, mas com interfaces padronizadas, e com o desenvolvimento de componentes externalizados para fornecedores, a IBM perdeu o controle sobre o conhecimento de arquitetura: este conhecimento ficou disperso entre os diferentes componentes e diferentes fornecedores.

Esse mesmo fenômeno ocorreu com a indústria de bicicletas, de acordo com o trabalho de Ulrich e Ellison (2004): a capacidade de inovar em produtos, hoje pertence às empresas que fabricam componentes como o câmbio e sistemas de freio (a Shimano, a "Intel das bicicletas"). A função de integrar os diferentes componentes em um único produto não é mais crítica para a inovação do produto, uma vez que a arquitetura de produto é modular, e seus componentes e interfaces são padronizados. 
Já no caso dos automóveis, não há uma correlação única entre funcionalidade e sua localização em um determinado módulo ou componente. Funções e características próprias de cada modelo como velocidade, conforto acústico, por exemplo, estão dispersas entre diversos módulos e componentes, o que faz com que o conhecimento de arquitetura seja fundamental na capacidade de inovar em produtos nesta indústria. Portanto, quem controla o conhecimento de arquitetura, possuiria a capacidade de inovar.

\section{Metodologia da pesquisa}

A externalização do DP no Brasil, como estratégia para obter vantagem competitiva sustentável, vem se intensificando desde os anos 1990 e seus impactos sobre a base de conhecimento das empresas ainda é recente e pouco conhecido. Além disso, é um processo complexo e lento, que envolve criação, dispersão e coordenação de conhecimento.

Por isso, pode-se afirmar que o estudo dos impactos da dispersão do conhecimento e de como as empresas que externalizam o DP estão lidando com isto, ainda está na fase de construção de teoria. O uso de estudo de caso como método de pesquisa é particularmente útil quando ainda não há certeza na definição dos constructos e variáveis que constroem a teoria que explicaria um fenômeno(VOSS et al,2002).

A pesquisa realizada neste trabalho será, portanto, de natureza qualitativa e será conduzida pelo método de estudo de caso. Os métodos de pesquisa qualitativa conduzidos através de estudo de caso são indicados para responder perguntas do tipo "como" e "por que" de um dado fenômeno (YIN, 2002).

O objeto de análise foi o processo de desenvolvimento de um veículo popular, especialmente no que se refere a estratégia de modularização e envolvimento de fornecedores. A empresa objeto do estudo foi a Volkswagen. O motivo da escolha está relacionado ao fato da empresa, em princípio, parecer apresentar uma área de desenvolvimento de produto sintonizada às necessidades dos clientes, o que posteriormente pôde ser observado pela boa aceitação de mercado do produto desenvolvido e pelo fato da empresa desenvolver localmente veículos.

Os módulos escolhidos para realização do estudo - cockpit e bancos - têm alto valor agregado, são tecnologicamente complexos (envolvem diferentes tipos de tecnologia na sua fabricação - como injeção de termoplásticos, soldagem, eletrônica, materiais têxteis, por exemplo), têm alto impacto na percepção da qualidade do produto pelos clientes e contam com envolvimento de fornecedores no seu desenvolvimento em variados graus no projeto objeto de estudo. 
Seguindo procedimentos propostos por Yin (2002) na coleta de dados, para o estudo do caso foram utilizadas três fontes de coleta de dados, a saber, documentação, entrevistas e observação direta.

Pode-se afirmar que as fontes de informação mais importantes utilizadas neste estudo foram as entrevistas. Utilizando-se questionários pré-estruturados, foram entrevistados individualmente executivos da montadora e das empresas fornecedoras do módulo banco e da cobertura do painel de instrumentos. Estes executivos pertencem às áreas de Desenvolvimento de Produto, Comercial e Qualidade. As entrevistas foram gravadas (quando permitidas pelo entrevistado) e depois transcritas na sua íntegra para melhor compreensão do material obtido.

A observação é outra forma de coletar evidências para o estudo de caso segundo Yin (2002). De uma maneira informal, foram realizadas observações diretas durante as visitas de campo para coletar evidências através de entrevistas. O levantamento observatório foi útil para fornecer informações adicionais sobre os tópicos do estudo.

\section{Estudo de Caso - O desenvolvimento do VW Fox}

Lançado no mercado nacional em 2003, o VW Fox foi inteiramente desenvolvido no Brasil, sobre a plataforma PQ 24 (do Polo), com a missão de ser um veículo de baixo custo, como forma da VW enfrentar a crescente concorrência no segmento. O Fox é fabricado nas fábricas de S.José dos Pinhais (juntamente com o Golf) e na fábrica da Anchieta (na nova linha do Polo). É também exportado para a América Latina e Europa.

\section{O módulo Cockpit - Projeto VW Fox}

O Cockpit é entregue como módulo diretamente na linha de montagem para o Fox. Na fábrica de São José dos Pinhais (onde são montados o Golf e o Fox mercado nacional), é montado e entregue por um fornecedor (o mesmo da Cobertura do Painel), localizado dentro da planta. Na fábrica Anchieta (para o Polo e Fox Exportação), é montado pela própria VW. Os componentes do módulo cockpit para o Fox apresenta-se na tabela abaixo: 


\begin{tabular}{|l|l|}
\hline \multicolumn{1}{|c|}{$\begin{array}{c}\text { Principais constituintes do } \\
\text { módulo cockpit }\end{array}$} & $\begin{array}{l}\text { Envolvimento de fornecedores } \\
\text { no desenvolvimento (para o } \\
\text { Fox) }\end{array}$ \\
\hline Suporte painel (Träger) & Co-design \\
\hline Cobertura painel & $\begin{array}{l}\text { Desenvolvimento do processo } \\
\text { de produção }\end{array}$ \\
\hline Caixa de ar & Black-box \\
\hline Módulo Air Bag & $\begin{array}{l}\text { Desenvolvimento do processo } \\
\text { de produção }\end{array}$ \\
\hline Volante & $\begin{array}{l}\text { Desenvolvimento de } \\
\text { ferramental }\end{array}$ \\
\hline Instrumento Combinado & Black-box \\
\hline Chicotes & Co-design \\
\hline Pedaleira & Co-design \\
\hline Coluna de direção & Co-design \\
\hline Diversas Fixações e Coberturas & $\begin{array}{l}\text { Desenvolvimento de } \\
\text { ferramental ou peças standard } \\
\text { (fixações) }\end{array}$ \\
\hline
\end{tabular}

Tabela 1 - Principais constituintes do módulo cockpit e responsabilidade no desenvolvimento Elaborada pela autora, a partir de dados da pesquisa

Dentre todas as peças, a mais crítica do ponto de vista de qualidade do acabamento, de custo e de montagem para o módulo como um todo, é a cobertura do painel. Ela deve absorver todas as variações de dimensão da carroceria e do suporte do painel, é uma peça de grandes dimensões, de complexa tecnologia de produção (envolve injeção de plástico, solda, montagem de componentes), com alto nível de exigência de qualidade visual (é a principal peça que o comprador sente e vê ao dirigir) e possui interfaces com peças de funcionalidade complexa (como o ar condicionado e o acionamento de todos comandos do motorista). Por esta razão, o desenvolvimento desse componente em particular, foi analisado em detalhes.

No desenvolvimento do módulo como um todo - detalhamento de quais peças o comporiam, quais especificações de interface (fixações na carroceria, p.ex) não houve nenhum envolvimento de fornecedores. O fornecedor do módulo para a planta de Curitiba tem contrato apenas de fornecimento de mão de obra e serviços logísticos e desenvolve apenas seu processo de produção.

Cada componente individual tem sua própria estratégia de envolvimento de fornecedores, dependendo de cada projeto (vide tabela 1). A caixa de ar e o instrumento combinado são blackboxes, ou seja, foram desenvolvidos totalmente por seus fornecedores a partir de um caderno de encargos ("Lastenheft"), que contêm as especificações de desempenho requeridas pela montadora. Outras peças, como o suporte do painel, foram desenvolvidas em co-design, ou seja, em parceria da montadora com o fornecedor, mas com a responsabilidade final sobre o projeto da montadora. $\mathrm{O}$ envolvimento dos fornecedores também pode ser através do desenvolvimento de ferramental (como no caso das coberturas plásticas), onde a montadora é responsável pelo conceito do produto, e o fornecedor pelo desenvolvimento do ferramental e demais meios de produção, ou através de 
desenvolvimento de processo, onde o fornecedor é envolvido apenas na fase desenvolvimento do processo de produção, sendo a montadora responsável pelo desenvolvimento do conceito do produto e de ferramental.

No caso do Fox, praticamente não houve envolvimento do fornecedor no desenvolvimento da cobertura do painel de instrumentos. Até o ferramental foi desenvolvido pela própria VW. A justificativa foi em função de custo e da definição tardia do fornecedor - em função de uma intensa discussão comercial, a escolha foi atrasada. O fornecedor apenas desenvolveu o processo de fabricação da peça. Muitos problemas de especificação da cobertura do painel só puderam ser verificados nesta fase, o que gerou necessidade de modificação de ferramental e consequentemente, aumento no custo de desenvolvimento e atrasos.

\section{O módulo Banco - Projeto VW Fox}

O conjunto de bancos é tratado como um módulo: o fornecedor é pago pela mão de obra empregada na montagem dos bancos, não havendo responsabilidade pelo desenvolvimento ou administração dos componentes. Ele é entregue sequenciado diretamente na linha de montagem. Para todas as unidades da VW, os bancos são fornecidos por terceiros. O módulo banco é composto pelos seguintes componentes:

\begin{tabular}{|l|l|}
\hline \multicolumn{1}{|c|}{$\begin{array}{c}\text { Principais constituintes do } \\
\text { módulo banco }\end{array}$} & $\begin{array}{l}\text { Envolvimento de fornecedores } \\
\text { no desenvolvimento (para o } \\
\text { Fox) }\end{array}$ \\
\hline Estrutura Metálica & Black box \\
\hline Espuma & $\begin{array}{l}\text { Desenvolvimento do processo } \\
\text { de produção }\end{array}$ \\
\hline Capa & $\begin{array}{l}\text { Desenvolvimento do processo } \\
\text { de produção }\end{array}$ \\
\hline Coberturas plásticas & $\begin{array}{l}\text { Desenvolvimento do } \\
\text { ferramental }\end{array}$ \\
\hline Apoio de Cabeça & $\begin{array}{l}\text { Desenvolvimento de } \\
\text { ferramental }\end{array}$ \\
\hline Fixações & Peças padronizadas \\
\hline
\end{tabular}

Tabela 2 - Principais constituintes do módulo banco e responsabilidade no desenvolvimento Elaborada pela autora, a partir de dados de pesquisa

No desenvolvimento do Fox, as especificações de interface entre os componentes que compõem o módulo foram todas determinadas pela montadora. O fornecedor do módulo não se envolveu no desenvolvimento do conjunto, nem de seus componentes nas fases iniciais do projeto; seu envolvimento com o processo foi apenas no desenvolvimento do processo de produção. Mas no desenvolvimento do banco, algumas especificações de componentes individuais só podem ser testadas após a montagem completa do módulo. Por este motivo, algumas modificações tiveram de ser realizadas no projeto dos componentes, acarretando sumento de custos para o produto. 
No caso dos componentes, o grau de envolvimento dos fornecedores é variado: há peças black-boxes, onde o projeto é de responsabilidade do fornecedor, como o caso das estruturas dos bancos dianteiros para o veículo 2 portas e dos bancos traseiros corrediços, peças onde o fornecedor desenvolveu o ferramental e onde somente desenvolveu o processo de produção.

Segundo o fornecedor entrevistado, é comum para outras montadoras instaladas aqui no Brasil como a GM e a Ford, o desenvolvimento do módulo como um todo pelos fornecedores.

\section{Análise dos Resultados e Conclusões}

Na descrição do desenvolvimento dos módulos banco e cockpit para o VW Fox, ficou claro que o controle das interfaces entre os diferentes componentes de cada módulo, que dão a funcionalidade integrada de cada um, ainda pertence à montadora.

Para os bancos, mesmo com componentes sendo desenvolvidos por fornecedores, é a VW quem determina o aspecto visual (cores, padronagens de tecidos), especificações funcionais, como torques e especificações de materiais (dureza de espuma, resistência a tração dos tecidos, resistência a radiação UV). O resultado é que características que determinam a percepção de qualidade do cliente tais como conforto e aspecto visual estão sob controle da montadora. Além disso, todos os testes funcionais para homologação do produto, como testes de durabilidade, segurança, rodagem em veículo, são feitos pela montadora. Mesmo quando ela não possui todos recursos internamente para realizar os testes (como testes de airbag em banco), utiliza-se os recursos do fornecedor, mas sob supervisão e especificação de parâmetros da montadora.

Também para as montadoras que externalizaram o desenvolvimento do banco completo, não há tanta liberdade para o fornecedor criar um produto inteiramente novo: as especificações básicas visuais e funcionais são determinadas pelas montadoras, que podem aceitar ou não, inovações e sugestões dos fornecedores.

Da mesma maneira, para o módulo cockpit, o controle das interfaces entre seus diferentes componentes pertence à montadora. Este módulo possui elevada complexidade funcional, integrando diferentes funções como painel de instrumentos, acionamento de diversos comandos do veículo e caixa de ar. Além disso, ele é fundamental para a percepção da qualidade dos clientes.

As palavras de um executivo do fornecedor de painel e módulo cockpit entrevistado ilustram bem o exemplo acima: 
[...] Cada montadora tem seu próprio conjunto de especificações sobre as quais elas não abrem mão, que são como dogmas, paradigmas de desenvolvimento muito fortes: o ponto $\mathrm{H}$, a altura do carro, o posicionamento do motorista, a distância entre o motorista e o painel, por exemplo - estas especificações são aquelas que o cliente identifica como característica própria de cada montadora e nós, fornecedores, desenvolvemos os componentes para atingir estas especificações.

A análise dos resultados obtidos nas entrevistas, análises de documentos e dados demonstram que o controle das interfaces e o conhecimento de como unir os diferentes módulos e componentes em um produto com funcionalidade integrada ainda pertence à montadora. $\mathrm{E} O$ domínio desse conhecimento faz com que a capacidade de inovar no mercado brasileiro de automóveis ainda pertença à montadora, mesmo com a tendência de externalizar atividades de DP para os fornecedores. Ficou claro, também, que as montadoras não mais possuem, ou desejam possuir, capacidade para desenvolver sozinhas um veículo completo, com toda sua complexidade tecnológica. As montadoras encontram-se dependentes em conhecimento, conforme o modelo de Fine e Whitney (1996), especialmente em tecnologias como a eletrônica.

Mas o fato de as montadoras estarem dependentes em conhecimento de seus fornecedores não implica diretamente em elas estarem perdendo a capacidade de inovar. Através da pesquisa bibliográfica e dos dados levantados, pode-se concluir que a manutenção da capacidade inovadora ao externalizar atividades de DP é influenciada pela manutenção do controle do conhecimento de arquitetura.

Contudo, é importante discutir o conceito de inovação em arquitetura, proposto por Henderson e Clark (1990). A inovação em arquitetura ocorre quando há uma reconfiguração de um sistema estabelecido de união dos componentes. Um exemplo seria o desenvolvimento de um ventilador portátil a partir de um ventilador de teto. Os componentes básicos do produto (pás, motor, sistemas de controle) permanecem o mesmo, mas a forma de configurá-los em um produto com diferentes requisitos de projeto (tamanho, localização do motor) faz com que as interações entre os componentes sejam alteradas, fazendo com que o conhecimento de arquitetura para o ventilador de teto seja inútil para desenvolver um ventilador portátil. A partir daí, pode-se questionar o que ocorreria com o conhecimento de arquitetura acumulado pelas montadoras se uma inovação, como o motor movido a hidrogênio, por exemplo, alterasse a arquitetura atual do veículo.

Por isso, pode-se concluir que o domínio do conhecimento de arquitetura garante a manutenção da capacidade de inovar dentro de um dado paradigma tecnológico - uma inovação em arquitetura pode alterar drasticamente o conhecimento de arquitetura necessário para desenvolver um novo produto. 
Para uma compreensão mais ampla deste tema, sugere-se no futuro uma pesquisa mais ampla sobre as conseqüências da externalização do DP em outras montadoras, em outros mercados com características diferentes do brasileiro e não só em montadoras de veículos, mas também em outros setores industriais, como o eletrônico, por exemplo.

Além disso, seria interessante verificar como outros fatores tais como a estratégia de produto da empresa, seu posicionamento na cadeia de suprimentos, sua trajetória e as características do setor e do mercado onde a empresa atua, por exemplo, podem afetar a manutenção da capacidade inovadora. O desenvolvimento de uma teoria sobre esse assunto seria muito importante para a pesquisa na área de Gestão de Tecnologia e Operações e também para praticantes do setor industrial.

\begin{abstract}
In order to diversify product portfolio and reduce development costs and timing, firms tend to outsource New Product Development (NPD). This could affect innovative capacity in two different ways: there could be significant technological contribution from supplier's technical expertise, or, in long range, could mean hollowing out its knowledge base. But how could firms enjoy benefits of NPD outsourcing without hollowing out? This paper attempts to discuss this question, analysing the impact of the Architectural Knowledge, or the knowledge about how to integrate different parts and modules into a coherent system, with integrated functionality, on maintenance of firm Innovative Capacity, with outsourcing of NPD. A cases study was conducted about the development of a new vehicle in Brazil. Conclusion suggests that Brazilian automakers are, so far, with current industry and product architecture structure, being able to maintain their Innovative Capacity even with their initiatives of outsourcing NPD.
\end{abstract}

Key-words: Innovative Capacity, Product Development, Automotive Industry.

\title{
Referências
}

BECKER, M. e ZIRPOLI, F. - Organizing New Product Development - Knowledge hollowing -out and knowledge integration - the Fiat Auto case - International Journal of Operations and Production Management: 2003 Vol.23, Nr.9

CLARK, K.B. e FUJIMOTO, T. Product Development Performance - Harvard Business School Press, 1991.

GRAZIADIO, T. e ZILBOVICIUS, M. - Knowledge transfer through the supply system: does modularity make it easier? - International Journal of Automotive Technology and Management. Vol. 3, no. 1-2, pp. 47-60. 2003 cross ${ }^{\text {ref }}$

FINE, C. e WHITNEY, D - Is the Make or Buy Decision a Core Competence?-IMVP/MIT Working Paper, Fev 1996

FIXSON, S. e SAKO,M. - Modularity in Product Architecture: Will the Auto Industry Follow the Computer Industry? (An Analysis of Product Architecture, Market Conditions and Industrial Forces) - IMVP/ MIT Working Paper, Set 2001 
HENDERSON, R.M. e CLARK, K. - Architectural Innovation: The Reconfiguration of Existing Product technologies and the Failure of Established Firms - Administrative Science Quarterly, 35, 1990

SAKO, M. - Modulariry and Outsourcing: The nature of Co-evolution of product Architecture and Organisation Architecture in the Global Automotive Industry - Cap. Livro - The Business of Systems Integrations, editado por Prencipe, Davies and Hobday - Oxford University Press, 2002

TAKEISHI, A. - Knowledge Partioning in the Interfirm Division of Labor - Organization Science - Vol.13, n³, MaiJun 2002.

ULRICH, K. e ELLISON, D. - Beyond Make-buy: Internalization and Integration of Design and Production Working Paper - Dept. Of Operations and Information Management - The Wharton School, University of Pennsylvania - Jan.2004.

VON HIPPEL, E. - The Sources of Innovation - Oxford University Press, New York, 1988

VOSS, C., TSIKRIKTSIS, N. E FROHLICH, M. - Case Research in Operations Management - International Journal of Operations and Production Management. Vol.22, n², pp-195-219. 2002

YIN, R. Estudo de caso: Planejamento e Métodos, Porto Alegre, Bookman, 2002.

\section{Dados dos autores:}

Adriana Marotti de Mello

Escola Politécnica da USP

Departamento de Engenharia de Produção

Av.Prof.Almeida Prado, trav.2, no.128 - Cidade Universitária - São Paulo - SP CEP 05508-070

Telefones para contato: 30915363 r.423

e-mail: adriana.mello@poli.usp.br

Roberto Marx

Escola Politécnica da USP

Departamento de Engenharia de Produção

Professor Doutor

Av.Prof.Almeida Prado, trav.2, no.128 - Cidade Universitária - São Paulo - SP CEP 05508-070

Telefones para contato: 30915363 r.423

e-mail:robemarx@usp.br 